\title{
The Group Evacuation Behavior Based on Fire Effect in the Complicated Three-Dimensional Space
}

\author{
Jun Hu, ${ }^{1,2}$ Huijun Sun, ${ }^{1}$ Ge Gao, ${ }^{1}$ Juan Wei, ${ }^{2}$ and Lei You ${ }^{3}$ \\ ${ }^{1}$ School of Traffic and Transportation, Beijing Jiaotong University, Beijing 100044, China \\ ${ }^{2}$ Faculty of Computer Science, Chengdu Normal University, Chengdu 611130, China \\ ${ }^{3}$ School of Electronic Engineering, University of Electronic Science and Technology of China, Chengdu 611731, China
}

Correspondence should be addressed to Juan Wei; weijuan0905@126.com

Received 25 November 2013; Revised 16 January 2014; Accepted 26 February 2014; Published 27 March 2014

Academic Editor: Baocang Ding

Copyright (C) 2014 Jun Hu et al. This is an open access article distributed under the Creative Commons Attribution License, which permits unrestricted use, distribution, and reproduction in any medium, provided the original work is properly cited.

\begin{abstract}
In order to effectively depict the group evacuation behavior in the complicated three-dimensional space, a novel pedestrian flow model is proposed with three-dimensional cellular automata. In this model the calculation methods of floor field and fire gain are elaborated at first, and the transition gain of target position at the next moment is defined. Then, in consideration of pedestrian intimacy and velocity change, the group evacuation strategy and evolution rules are given. Finally, the experiments were conducted with the simulation platform to study the relationships of evacuation time, pedestrian density, average system velocity, and smoke spreading velocity. The results had shown that large-scale group evacuation should be avoided, and in case of large pedestrian density, the shortest route of evacuation strategy would extend system evacuation time.
\end{abstract}

\section{Introduction}

As the density of urban population increases rapidly, the sudden disaster in the complicated environment may bring about serious consequences, so the study focus of the pedestrian flow is how to provide effective evacuation strategy [1-5]. At present, the evacuation methods of pedestrian flow are mainly divided into continuous model and discrete model. The typical continuous model is social force, which was used by Helbing et al. [6] to study evacuation process of pedestrians, discovering the formation mechanism of phenomena such as "faster is slower" and "arching" effect. The typical discrete model is lattice gas and cellular automata. The pedestrian evacuation efficiency in environment such as bottleneck channel, T-type channel, and hall was studied by Tajima et al. [7-9] with lattice gas model, analyzing the critical threshold value with phase transformation under different conditions. The evacuation process of pedestrian flow was studied by Guo and Huang [10] with dynamic lattice gas model. Through considering the repulsive force in cellular automata, Kirchner et al. [11, 12] studied the phenomena of pedestrians avoiding each other when competing for the same position. The pedestrian evacuation model of twodimensional cellular automata was established by Burstedde et al. [13], which proposed "floor field" concept to study typical phenomenon. Nagai et al. [14] studied the evacuation characteristics of pedestrians whose view is blocked completely.

Moreover, the pedestrians' intimacy often leads to group evacuation. Based on $\mathrm{A}^{*}$ algorithm, the evacuation efficiency of different-scale group was studied by Ji and Gao [15] through establishing the group evacuation model with leader who determined the direction of group evacuation. Qiu and $\mathrm{Hu}$ [16] established evacuation model and depicted the group behavior for evacuation efficiency, and the simulation results showed that the congestion degree was influenced by group scale. By combining conformity mentality, Low [17] elaborated the phenomena of some group following another group in evacuation. Furthermore, the factors such as pedestrians' panic $[18,19]$, game [20], interaction between pedestrians [21], and fire spreading [22] were also introduced to cellular automata model to study evacuation efficiency.

Meanwhile, pedestrians' velocity of walking upward or downward the stairway is clearly different from their velocity 


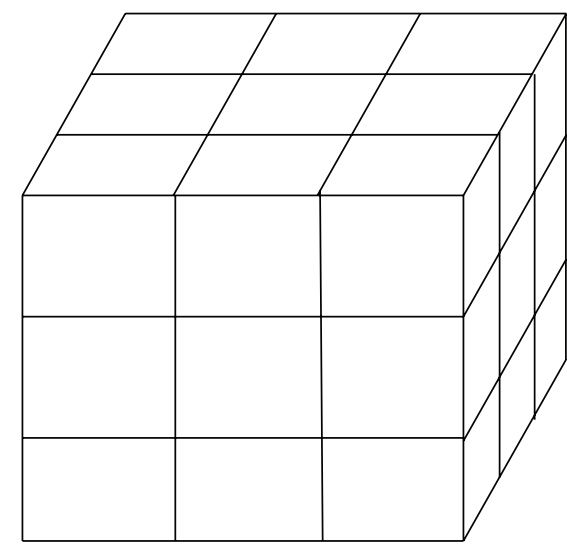

(a) Cellular structure

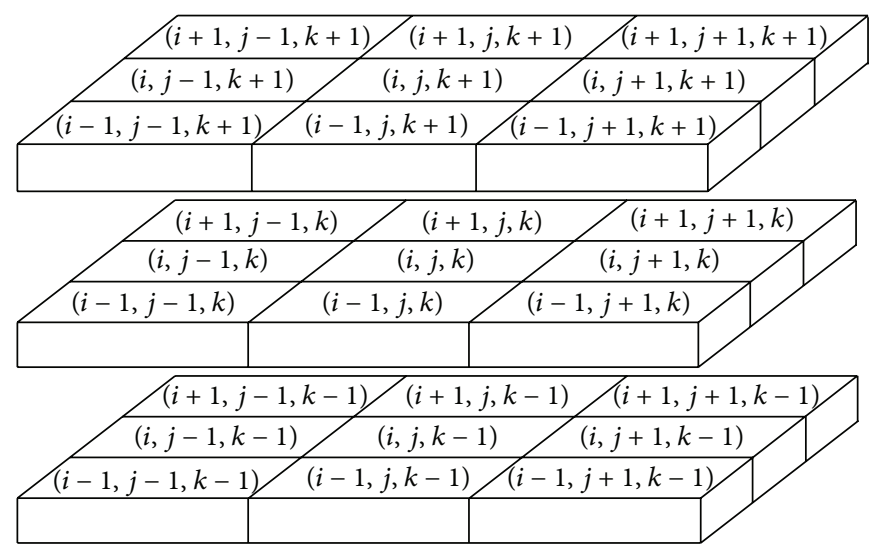

(b) Position schematic diagram

Figure 1: The three-dimensional Moore structure.

of walking horizontally, which has great effect on the evacuation efficiency. Kretz et al. [23] found that the distribution of horizontal walking velocity exhibited a maximum between $0.4 \mathrm{~m} / \mathrm{s}$ and $0.5 \mathrm{~m} / \mathrm{s}$ in the local density. And some pedestrians actually accelerate when walking upward a short stairway; however, such a phenomenon could neither be observed in downward motion on the short stairway nor in upward motion on the long stairway. However, the presented cellular automata model mainly focuses on the two-dimensional platform, which is different from the actual three-dimensional evacuation space containing stairways. But stairway factor has a great influence on the evacuation velocity and evacuation efficiency. Therefore, in this thesis a novel cellular automata model is proposed for the three-dimensional space in the complicated environment, and the group behavior is studied with different evacuation strategies when fire breaks out.

\section{Methodology}

As shown in Figure 1, the two-dimensional Moore structure is extended to the three-dimensional structure, every cellular of which is a basic unit. There are three statuses for every cellular: occupied by pedestrian, occupied by obstacle, or vacant. It is assumed that the pedestrian is located at the cellular $(i, j, k)$; in each time step, the transition gain $Q_{i j k}$ of the neighboring twenty-six positions and his own position is calculated by (1), making the smallest transition gain as the target position at the next moment:

$$
Q_{i j k}=\left(\alpha S_{i j k}+\beta F_{i j k}\right)\left(1-n_{i j k}\right)\left(1-\varphi_{i j k}\right) \sigma_{i j k} \xi_{i j k} .
$$

In this formula, $i, j$, and $k$ are the position coordinates, $Q_{i j k}$ is the transition gain of cellular $(i, j, k) ; n_{i j k}$ is the vacancy coefficient; if cellular $(i, j, k)$ has been occupied by other pedestrians, then $n_{i j k}=1$; otherwise $n_{i j k}=0$; $\xi_{i j k}$ is the obstacle coefficient, if cellular $(i, j, k)$ is the obstacle, then $\xi_{i j k}=0$; otherwise $\xi_{i j k}=1 ; S_{i j k}$ and $\alpha$ indicate the floor field and its influence coefficient; the bigger $S_{i j k}$ is, the further it is from the exit; $F_{i j k}$ and $\beta$ indicate the fire gain and its influence coefficient, the bigger $F_{i j k}$ is, the more serious it is influenced by fire. And $\alpha+\beta=1,0 \leq \alpha \leq 1,0 \leq \beta \leq 1$; the bigger $\alpha$ is, the more possible it is for pedestrians to adopt the shortest route strategy; the bigger $\beta$ is, the more possible it is for pedestrians to consider avoiding being influenced by fire.

Meanwhile, different from the two-dimensional cellular automata Moore structure, the parameter $\varphi_{i j k}$ is added to indicate whether the cellular $(i, j, k)$ exists or not. If cellular $(i, j, k)$ does not exist, then $\varphi_{i j k}=1$; otherwise $\varphi_{i j k}=0$. And the parameter $\sigma_{i j k}$ is used to indicate the transition gains for walking upstairs, downstairs, or horizontally. Usually, the pedestrians tend to walk horizontally more, which saves physical power best, and then the second best is walking downward the stairways; the last is walking upward the stairways. Therefore, the parameter $\sigma_{i j k}$ is set as

$$
\sigma_{i j k}= \begin{cases}1.5, & \text { for walking horizontally, } \\ 1, & \text { for walking downstairs, } \\ 0.5, & \text { for walking upstairs. }\end{cases}
$$

2.1. The Floor Field. In the two-dimensional platform, the calculation methods of "floor field" have been put forward in many studies [11-13, 24-26]. In this thesis, the calculation method of floor field is proposed in three-dimensional space. Considering the complicated three-dimensional space as shown in Figure 2, there exists an exit (the width of which is five cellular), and the white areas stand for wall or obstacle. The floor field $S_{i j k}$ of the cellular $(i, j, k)$ is determined based on the following principles: (1) for the wall or obstacle, the floor field $S_{i j k}=500$, indicating that the pedestrians cannot pass; (2) for the exit, the floor field $S_{i j k}=0$; (3) the floor field of the straight adjacent cellular at the same level as the exit is added by 1 , the floor field of the diagonal adjacent cellular at the same level as the exit is added by $\sqrt{2}$, the floor field of the straight adjacent cellular at the adjacent level as the exit is added by $\sqrt{2}$, and the floor field of the diagonal adjacent cellular at the adjacent level as the exit is added by $\sqrt{3}$; (4) the floor field of other cellular is determined according to (3). 


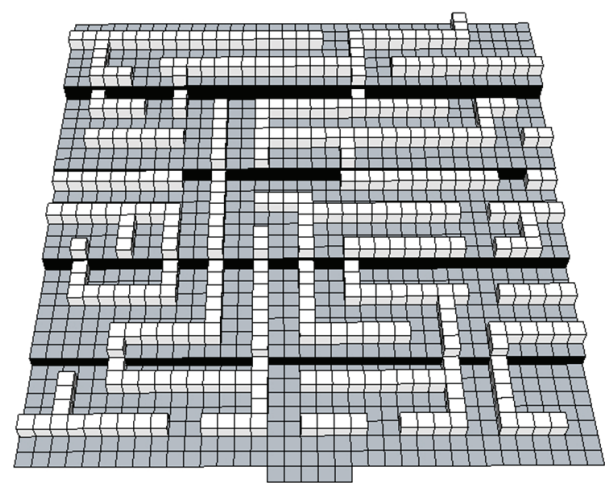

FIgURE 2: Three-dimensional space.

Based on the principles above, the floor field of threedimensional space is shown in Figure 3:

$$
S_{i j k}=\left\{\begin{array}{c}
\min \left(S_{(i-1) j k}+1, S_{i(j-1) k}+1,\right. \\
\left.\quad S_{(i-1)(j-1) k}+\sqrt{2}\right), \\
\text { if in the same layer } \\
\min \left(S_{(i-1) j k}+1, S_{i(j-1)(k-1)}+\sqrt{2},\right. \\
\left.\quad S_{(i-1)(j-1)(k-1)}+\sqrt{3}\right), \\
\quad \text { if in the different layer } \\
0, \quad \text { if }(i, j, k) \text { exists } \\
500, \quad \text { if }(i, j, k) \text { is wall or obstacle. }
\end{array}\right.
$$

2.2. The Fire Gain. Usually, after the fire breaks out, the spreading velocity of smoke has major impact on evacuation. For the sake of depicting the problems clearly, the influence of smoke on the cellular $(i, j, k)$ is divided into three grades: no effect, general effect, and serious effect. If the cellular $(i, j, k)$ is the fire source or influenced seriously, then the fire gain $F_{i j k}=500$, indicating that pedestrians cannot pass; if the cellular $(i, j, k)$ is not influenced by the smoke, then $F_{i j k}=0$. The fire gain $F_{i j k}$ is shown in

$$
F_{i j k}= \begin{cases}0, & \text { for no effect, } \\ \lambda, & \text { for general effect, } \\ 500, & \text { for serious effect or fire source. }\end{cases}
$$

In this formula, $\lambda(\lambda>0)$ is the fire coefficient. It is assumed that the cellular $\left(i_{f}, j_{f}, k_{f}\right)$ is the fire source, which spreads to the neighboring cellular after $2 m$ time steps. The influence of the smoke exerted on the cellular $(i, j, k)$ is set to be general effect initially; then it is changed to serious effect after $14 m$ time steps. The smoke continues to spread until the whole evacuation space is evacuated.

According to the findings of [23], pedestrians' velocity of walking horizontally on the stairways $v=0.42 \mathrm{~m} / \mathrm{s}$, the average velocity of walking upstairs $v=0.71 \mathrm{~m} / \mathrm{s}$, and the average velocity of walking downstairs $v=0.65 \mathrm{~m} / \mathrm{s}$. But in reality, when influenced by the smoke, the pedestrians' walking velocity would be slower. The pedestrians' walking velocity under influence of the smoke is set as

$$
v= \begin{cases}v, & \text { for no effect } \\ \frac{v}{\lambda}, & \text { for general effect } \\ 0, & \text { for serious effect or fire source }\end{cases}
$$

2.3. Group Behavior. As influenced by intimacy and conformity mentality, under emergency and in the complicated environment, pedestrians tend to evacuate in herding. Therefore, in this thesis, the effect of group behavior on the evacuation efficiency is studied. Assume group $G$ is formed; the location of group becomes the key factor of characterization. As the location information of group has some range, for the convenience of depiction, the average location of all members is defined as the location of group GLocation $(i, j, k)$. But we should exclude the fact that some members are far from the group, for it makes the central location of the group not the actual center. The location coordinates of all members in the group are sorted based on axes $i, j$, and $k$, obtaining three sorted arrays $\left(i_{1}, i_{2}, \ldots, i_{\mathrm{NUM}}\right),\left(j_{1}, j_{2}, \ldots, i_{\mathrm{NUM}}\right)$, and $\left(k_{1}, k_{2}, \ldots, k_{\mathrm{NUM}}\right)$, and NUM is the number of group. Then $\phi \%$ (generally, $5<\phi<20)$ data in front and back of every array are dislodged, the group center GLocation $(i, j, k)$ is calculated according to (6) with the remaining data num $($ num $=\operatorname{NUM}(1-\phi \%))$. This is to avoid the impact of some far away members exerted on the group central location:

$$
\begin{aligned}
& i=\left\lfloor\frac{1}{\text { num }} \sum_{X=1}^{\text {num }} i_{X}\right\rfloor, \quad j=\left\lfloor\frac{1}{\text { num }} \sum_{X=1}^{\text {num }} j_{X}\right\rfloor, \\
& k=\left\lfloor\frac{1}{\text { num }} \sum_{X=1}^{\text {num }} k_{X}\right\rfloor .
\end{aligned}
$$

In this formula, $L\rfloor$ indicates the rounding down. Meanwhile, the biggest distance between the location of member $X$ Location $\left(i_{X}, j_{X}, k_{X}\right)$ and the group center GLocation $(i, j, k)$ is defined as $D$, if

$$
\sqrt{\left(i_{X}-i\right)^{2}+\left(j_{X}-j\right)^{2}+\left(k_{X}-k\right)^{2}}>D .
$$

Then, the member $X$ should immediately adjust the current position by moving to group center GLocation $(i, j, k)$ at once.

2.4. Evolution Rule. The model adopts parallel update mechanism, and the pedestrians meet the following rules from moment of $t$ to moment of $t+1$ in evacuation.

(1) At the initial moment, the groups are initialized based on intimacy; the members of group calculate the transition gain $Q_{i j k}$ of the alternative position at moment of $t+1$ according to (1). Meanwhile, the smallest transition gain $Q_{i j k}$ is set as the target 


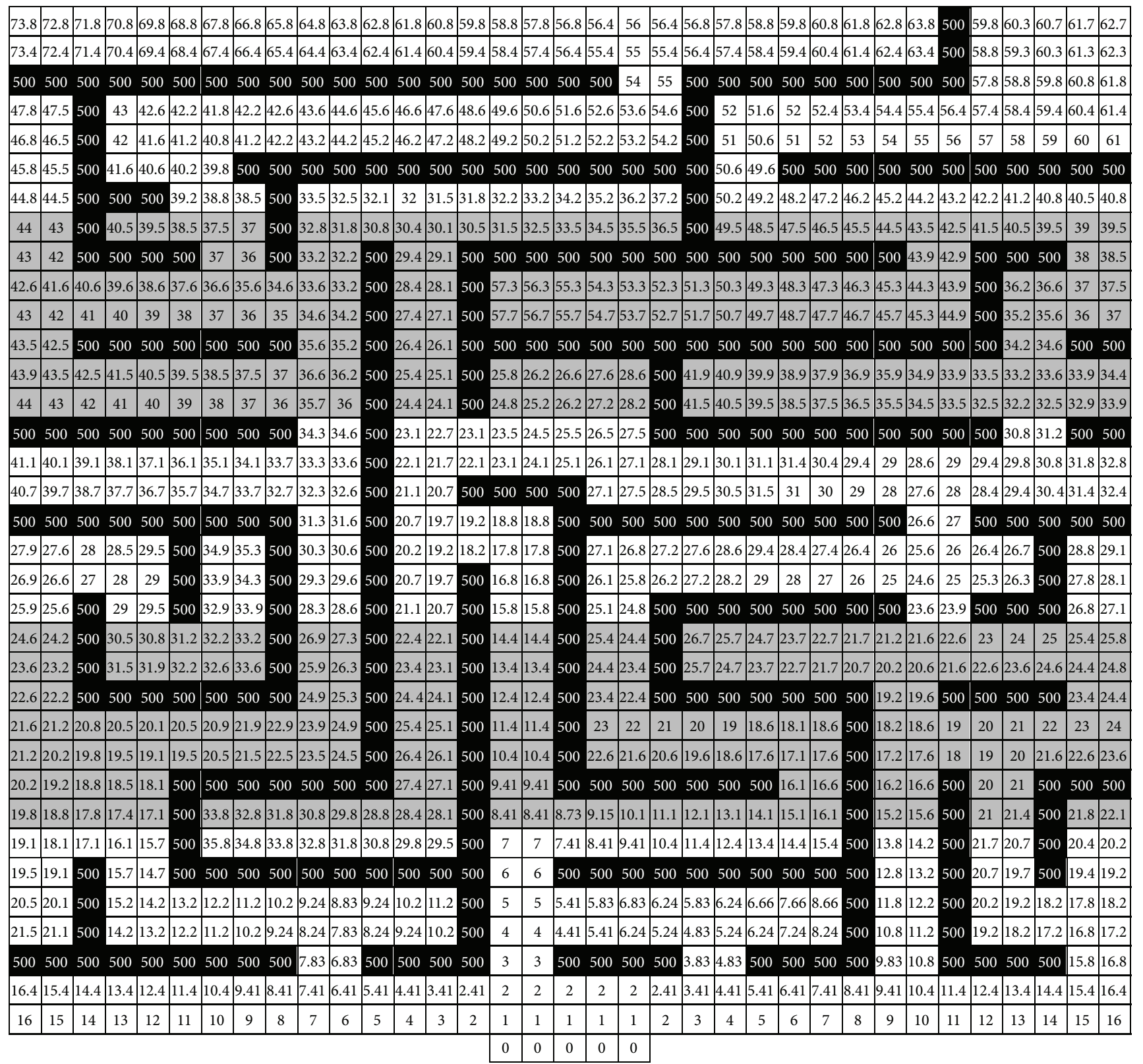

Figure 3: The floor field.

position at moment of $t+1$. If there exist many identical smallest transition gains, one position is randomly chosen with equal probability.

(2) According to (6) and (7), if the distance of some group member with the GLocation $(i, j, k)$ exceeds maximum $D$, the group member should immediately adjust the current position by moving to group center GLocation $(i, j, k)$.

(3) When many pedestrians compete for one target position at the same time, one of the pedestrians is chosen randomly by the system with equal probability to move to the target position, and the other pedestrians stay still.
(4) The pedestrians would evacuate out of the system at moment of $t+1$ when moving to the exit.

(5) When all pedestrians finish evacuating, the simulation is completed.

\section{Experiment}

3.1. Simulation. Based on the three-dimensional automata model above, the experiment platform is established to study the performance of group evacuation when the fire breaks out. The complicated three-dimensional evacuation space is considered as shown in Figure 2. The volume of evacuation space is set as $14 \mathrm{~m} \times 14 \mathrm{~m} \times 1 \mathrm{~m}(1 \mathrm{~m}$ is the height from the first floor to the last floor), and there are five floors and 


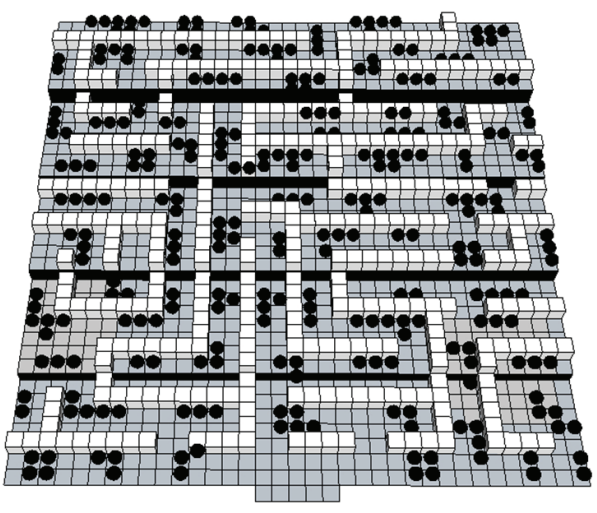

(a) $t=5$

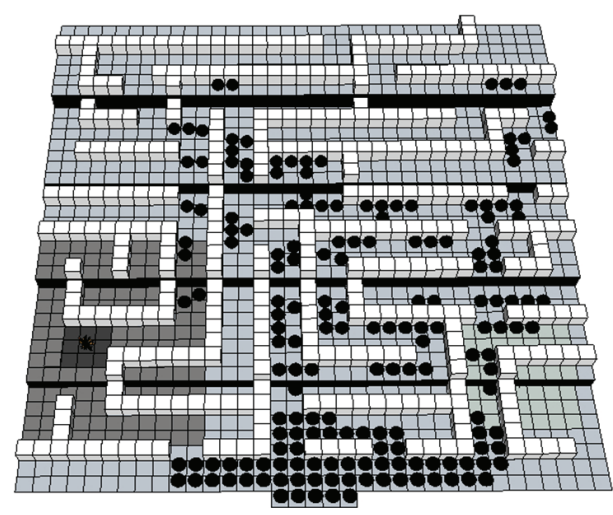

(b) $t=58$

FIGURE 4: The evacuation process.

an exit. The platforms are dispersed as cellular of $0.4 \mathrm{~m} \times$ $0.4 \mathrm{~m} \times 0.2 \mathrm{~m}$ (in which, $0.2 \mathrm{~m}$ is the height of stair); every cellular (for platform with $0.4 \mathrm{~m} \times 0.4 \mathrm{~m}$ ) can hold only one pedestrian, and the width of the exit is set as five cellular. On experiment platform, the black ball indicates pedestrian, the white area indicates obstacle or wall (the floor field $S_{i j k}=$ 500 ), and the grey area indicates smoke; in every unit time step pedestrians can only move to the neighboring position or stay still. Meanwhile, pedestrian density $\rho$ is defined as the ratio of the number of pedestrians to the number of all cellular in evacuation space, average system velocity $V$ is defined as the average velocity of all pedestrians, and system evacuation time $T$ is defined as the time for all pedestrians to leave the evacuation. It is set as $\rho=0.3, \alpha=\beta=0.5, D=$ $3, \lambda=2, \phi=15$, and $m=2$. The fire source is set on the left side; the smoke is produced when $t=30 \mathrm{~s}$. In Figure 4 , the evacuation under the different time step is shown. When $t=$ $5 \mathrm{~s}$, the pedestrians are distributed evenly in the evacuation space. After the fire breaks out, the pedestrians choose the middle part or the right side for evacuation to avoid the fire source or smoke. Moreover, the "arching" effect at the exit is consistent with the reality

3.2. Results and Discussion. By combining the above initial state and experimental results, the discussion of performance is made. In order to reduce the impact of the initial experiment status or waiting probability on the statistical indicators, the average value of thirty runtime value is taken for every indicator. In Figure 5, the relationship between system evacuation time $T$ and the number of group members NUM under the different parameter $\beta$ is given. From Figure 5 it can be seen that as NUM increases, system evacuation time $T$ tends to increase as well; that is to say, the larger the scale of group, the more harmful it is to improve the evacuation efficiency. When NUM is small, the smaller the parameter $\beta$, the shorter the system evacuation time. But when NUM is large, the curve suddenly changes: the bigger the parameter $\beta$, the shorter the system evacuation time. This indicates that when other factors are unchanged, the small group adopting the shortest route strategy is good for improving evacuation efficiency, while large-scale group

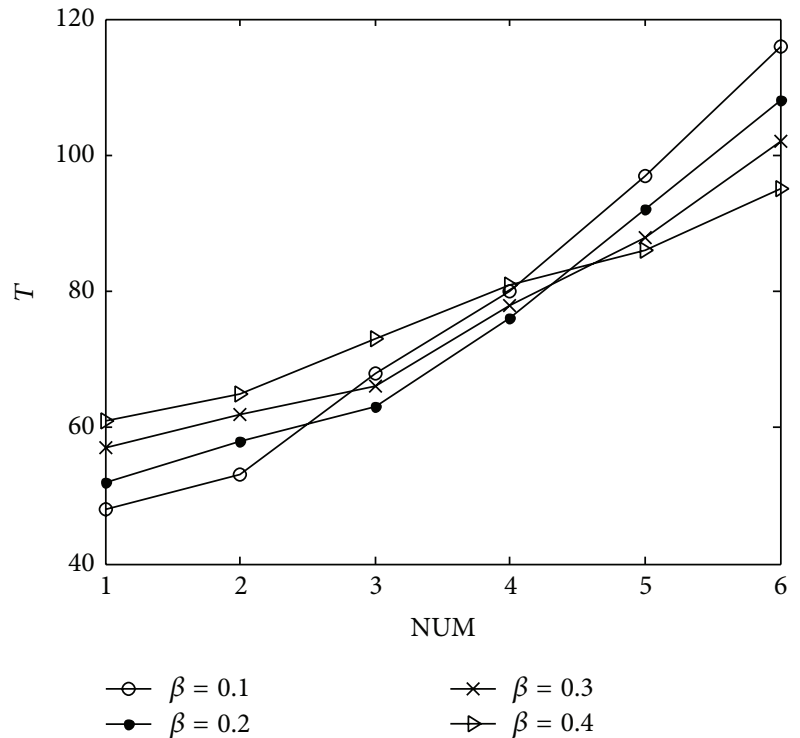

FIGURE 5: The relationship between system evacuation time $T$ and the number of group members NUM.

who choose to keep away from the fire is more beneficial for improving evacuation efficiency. It reflects that when the group scale is constant, the best evacuation efficiency may be achieved with the balanced evacuation method. Furthermore, in Figure 6 the relationship between system evacuation time $T$ and pedestrian density $\rho$ under the different parameter $\beta$ is given. Similar to Figure 5, there occurs sudden change of curve in Figure 6. When pedestrian density is small, the smaller the parameter $\beta$, the shorter the system evacuation time. But when pedestrian density is large, the bigger the parameter $\beta$, the smaller the evacuation time. In case of large pedestrian density, if the shortest route strategy is preferred for evacuation, the congestion may be produced as the smoke would block some part of evacuation routes, which extends system evacuation time.

Meanwhile, the relationship between system evacuation time $T$ and average system velocity $V$ is shown in Figure 7 . 


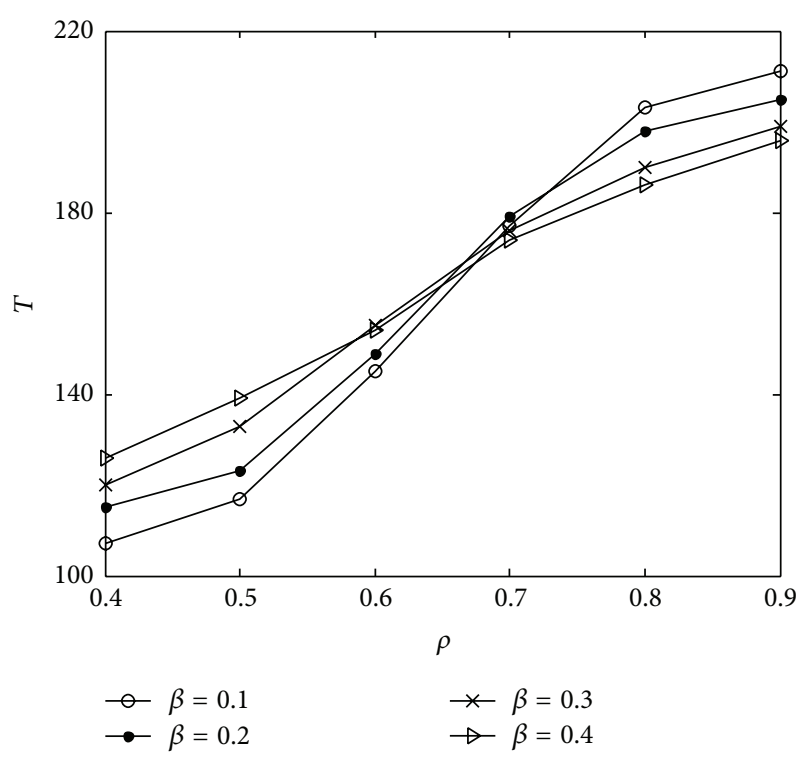

FIGURE 6: The relationship between system evacuation time $T$ and pedestrian density $\rho$.

From Figure 7, as average system velocity $V$ increases, system evacuation time $T$ tends to decrease firstly and increase secondly, and the inflection of curve emerges when $V=$ 1.8. This indicates that it cannot reduce the evacuation time by improving the evacuation velocity only. As the whole evacuation velocity of the pedestrians improves, serious congestion may be created at the exit, which is consistent with the "faster is slower" effect [19].

Finally, the relationship between system evacuation time $T$ and the time step of smoke spreading velocity $m$ under the different parameter $\beta$ is given in Figure 8 . The smaller the $m$, the faster the smoke spreading. From the figure it can be seen that as $m$ increases, system evacuation time $T$ tends to decrease. The smaller the impact of smoke exerted on the evacuation space, the higher the evacuation efficiency. Meanwhile, when $m$ is smaller, system evacuation time corresponding to the shortest route strategy (such as $\beta=0.1$ ) shows longer evacuation time. Under such condition, that is, the smoke spreading velocity is big, it is bad for reducing system evacuation time to adopt the shortest route strategy. When $m$ is big, the smoke spreading velocity is small; it can improve the evacuation efficiency to adopt the shortest route strategy (such as $\beta=0.1$ ).

\section{Conclusions}

In order to depict the group evacuation behavior and evacuation efficiency when fire breaks out in the complicated threedimensional environment, a novel evacuation model is established based on the three-dimensional cellular automata. The calculation methods of floor field and fire gain are given in the model, in which the transition gain of the target position at the next moment is defined, and pedestrians will move to the position which is the minimum gain. Meanwhile, based on pedestrians' intimacy and velocity change, the group

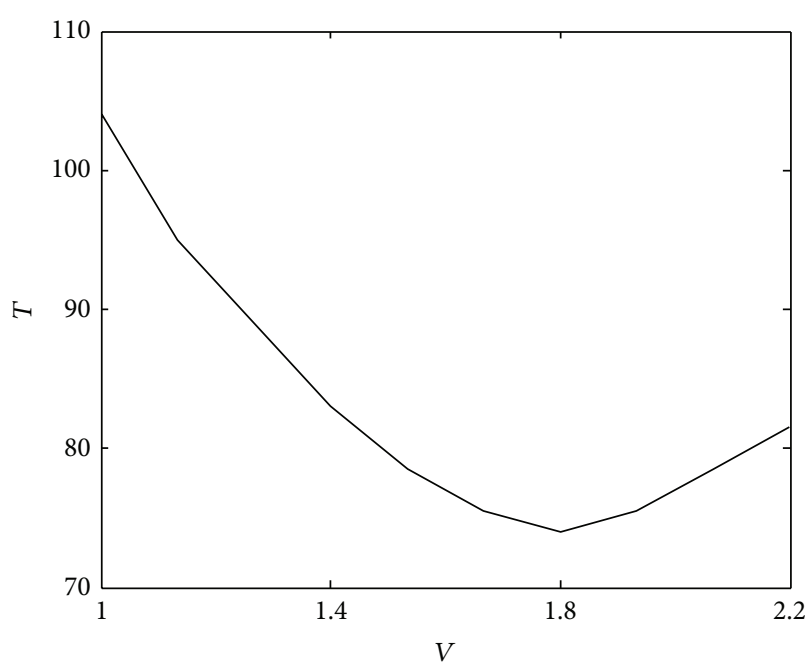

FIGURE 7: The relationship between system evacuation time $T$ and average system velocity $V$.

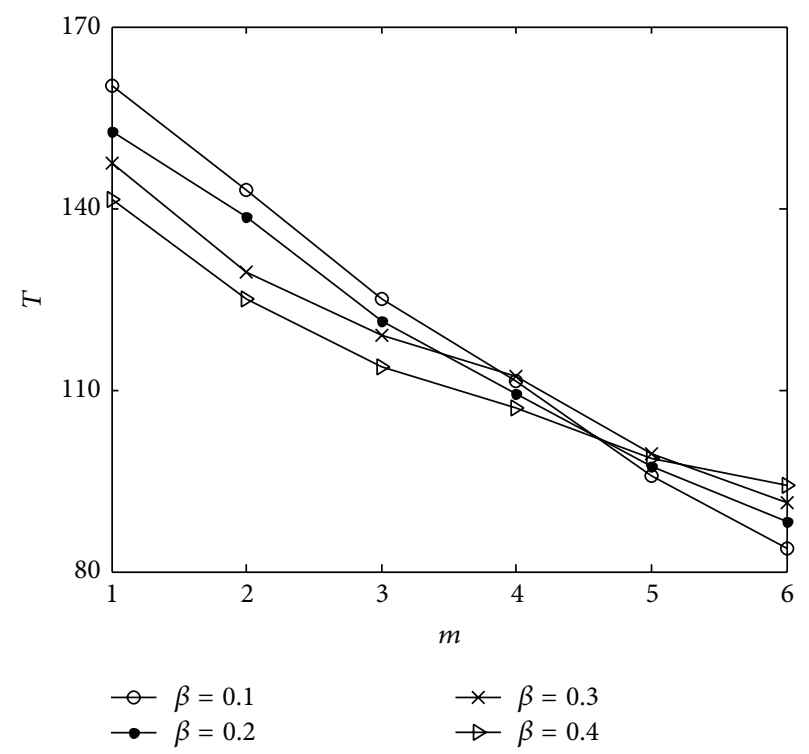

FIGURE 8: The relationship between system evacuation time $T$ and the time step of smoke spreading $m$.

evacuation strategy is elaborated. At last, the established experiment platform is used for simulation; the results show that (1) in case of large pedestrian density, the shortest route strategy would extend the system evacuation time; (2) the larger scale the group, the lower the evacuation efficiency; (3) it cannot effectively reduce the evacuation time by improving the evacuation velocity only. In our future studies, the panic mentality and the interaction of pedestrians would be taken into account in the three-dimensional automata model.

\section{Conflict of Interests}

The authors declare that there is no conflict of interests regarding the publication of this paper. They also would like 
to thank the reviewers for their valuable comments on the paper.

\section{Acknowledgments}

This research was supported by the National Natural Science Foundation of China (Grant no. 61304187), the Program for New Century Excellent Talents in University of China (Grant no. NCET-12-0764), the Scientific Research Fund of Sichuan Provincial Science and Technology Department (Grants nos. 2014JY0111, 2013GZ0016), the Scientific Research Foundation of the Education Department of Sichuan Province of China (Grant no. 13ZA0296, 13ZB0052), and the Funds of Chengdu Normal University of China (Grants nos. CS13ZD01, YJRC2012-6).

\section{References}

[1] G. A. Frank and C. O. Dorso, "Room evacuation in the presence of an obstacle," Physica A, vol. 390, no. 11, pp. 2135-2145, 2011.

[2] J. S. Michael and K. Gerta, "Natural discretization of pedestrian movement in continuous space," Physical Review E, vol. 86, no. 4, Article ID 046108, 2012.

[3] E. Takahiro, Y. Daichi, and N. Katsuhiro, "Pedestrian flow through multiple bottlenecks," Physical Review E, vol. 86, no. 2, Article ID 026118, 2012.

[4] J. Izquierdo, I. Montalvo, R. Pérez, and V. S. Fuertes, "Forecasting pedestrian evacuation times by using swarm intelligence," Physica A, vol. 388, no. 7, pp. 1213-1220, 2009.

[5] G. Baglietto and D. R. Parisi, "Continuous-space automaton model for pedestrian dynamics," Physical Review E, vol. 83, no. 5, Article ID 056117, 2011.

[6] D. Helbing, F. Schweitzer, J. Keltsch, and P. Molnár, "Active walker model for the formation of human and animal trail systems," Physical Review E, vol. 56, no. 3, pp. 2527-2539, 1997.

[7] Y. Tajima, K. Takimoto, and T. Nagatani, "Scaling of pedestrian channel flow with a bottleneck," Physica A, vol. 294, no. 1-2, pp. 257-268, 2001.

[8] Y. Tajima and T. Nagatani, "Clogging transition of pedestrian flow in T-shaped channel," Physica A, vol. 303, no. 1-2, pp. 239250, 2002.

[9] Y. Tajima and T. Nagatani, "Scaling behavior of crowd flow outside a hall," Physica A, vol. 292, no. 1-4, pp. 545-554, 2001.

[10] R. Y. Guo and H. J. Huang, "A mobile lattice gas model for simulating pedestrian evacuation," Physica A, vol. 387, no. 2-3, pp. 580-586, 2008.

[11] A. Kirchner and A. Schadschneider, "Simulation of evacuation processes using a bionics-inspired cellular automaton model for pedestrian dynamics," Physica A, vol. 312, no. 1-2, pp. 260-276, 2002.

[12] A. Kirchner, K. Nishinari, and A. Schadschneider, "Friction effects and clogging in a cellular automaton model for pedestrian dynamics," Physical Review E, vol. 67, no. 5, Article ID 056122, 2003.

[13] C. Burstedde, K. Klauck, A. Schadschneider, and J. Zittartz, "Simulation of pedestrian dynamics using a two-dimensional cellular automaton," Physica A, vol. 295, no. 3-4, pp. 507-525, 2001.

[14] R. Nagai, T. Nagatani, M. Isobe, and T. Adachi, "Effect of exit configuration on evacuation of a room without visibility," Physica A, vol. 343, no. 1-4, pp. 712-724, 2004.
[15] Q. G. Ji and C. Gao, "Simulating crowd evacuation with a leaderfollower model," International Journal of Computer Sciences and Engineering Systems, vol. 1, no. 4, pp. 249-252, 2007.

[16] F. Qiu and X. Hu, "Modeling group structures in pedestrian crowd simulation," Simulation Modelling Practice and Theory, vol. 18, no. 2, pp. 190-205, 2010.

[17] D. J. Low, "Statistical physics: following the crowd," Nature, vol. 407, no. 6803, pp. 465-466, 2000.

[18] D.-F. Xie, Z.-Y. Gao, X.-M. Zhao, and D. Z.-W. Wang, "Agitated behavior and elastic characteristics of pedestrians in an alternative floor field model for pedestrian dynamics," Physica A, vol. 391, no. 7, pp. 2390-2400, 2012.

[19] D. Helbing, I. Farkas, and T. Vicsek, "Simulating dynamical features of escape panic," Nature, vol. 407, no. 6803, pp. 487490, 2000.

[20] J. Tanimoto, A. Hagishima, and Y. Tanaka, "Study of bottleneck effect at an emergency evacuation exit using cellular automata model, mean field approximation analysis, and game theory," Physica A, vol. 389, no. 24, pp. 5611-5618, 2010.

[21] S. Wei-Guo, Y. Yan-Fei, W. Bing-Hong, and F. Wei-Cheng, "Evacuation behaviors at exit in CA model with force essentials: a comparison with social force model," Physica A, vol. 371, no. 2, pp. 658-666, 2006.

[22] Y. Zheng, B. Jia, X.-G. Li, and N. Zhu, "Evacuation dynamics with fire spreading based on cellular automaton," Physica A, vol. 390, no. 18-19, pp. 3147-3156, 2011.

[23] T. Kretz, A. Grünebohm, A. Kessel, H. Klüpfel, T. Meyer-König, and M. Schreckenberg, "Upstairs walking speed distributions on a long stairway," Safety Science, vol. 46, no. 1, pp. 72-78, 2008.

[24] T. Kretz, A. Große, S. Hengst, L. Kautzsch, A. Pohlmann, and P. Vortisch, "Quickest paths in simulations of pedestrians," Advances in Complex Systems, vol. 14, no. 5, pp. 733-759, 2011.

[25] A. Kirchner, H. Klüpfel, K. Nishinari, A. Schadschneider, and M. Schreckenberg, "Simulation of competitive egress behavior: comparison with aircraft evacuation data," Physica A, vol. 324, no. 3-4, pp. 689-697, 2003.

[26] A. Varas, M. D. Cornejo, D. Mainemer et al., "Cellular automaton model for evacuation process with obstacles," Physica A, vol. 382, no. 2, pp. 631-642, 2007. 


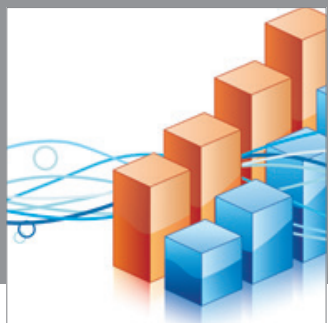

Advances in

Operations Research

mansans

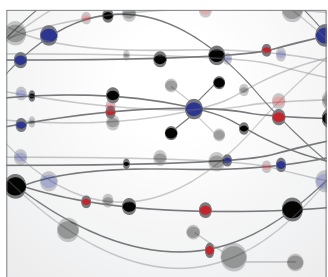

The Scientific World Journal
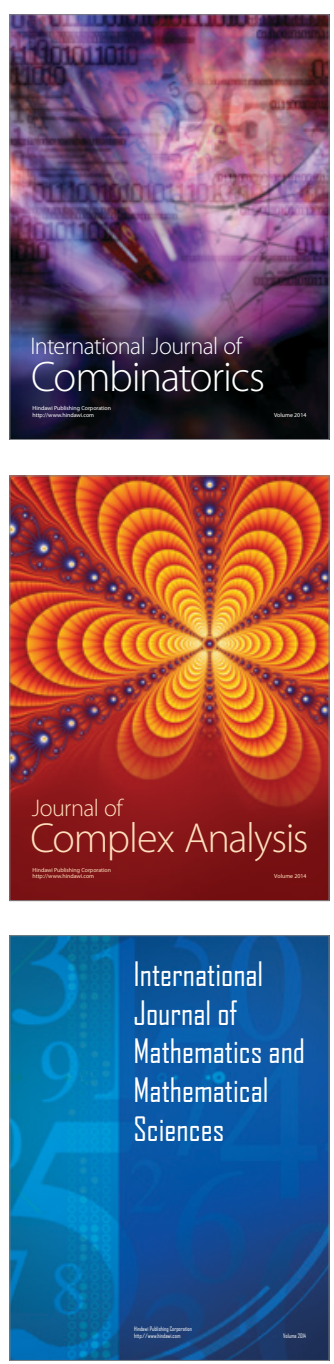
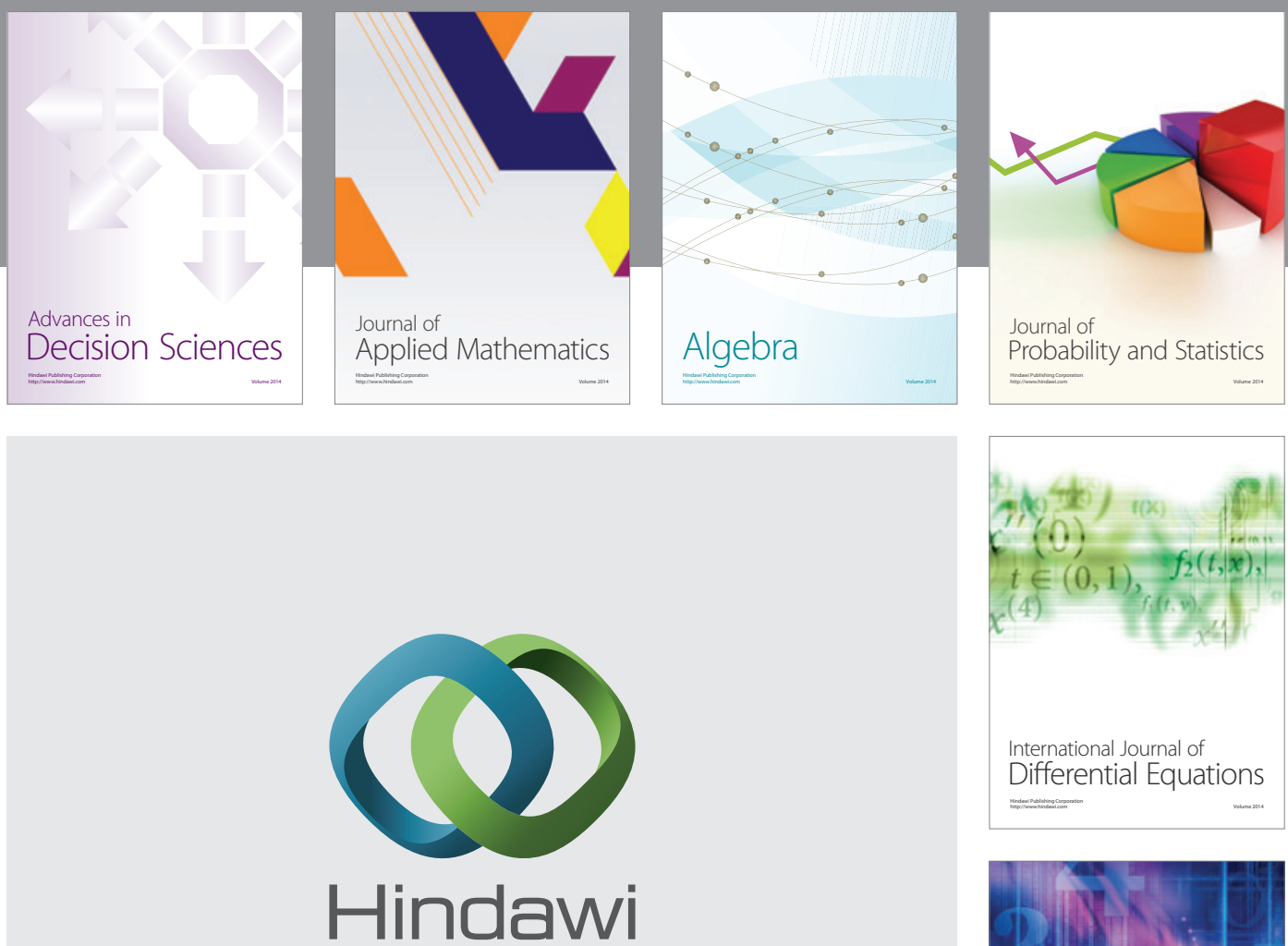

Submit your manuscripts at http://www.hindawi.com
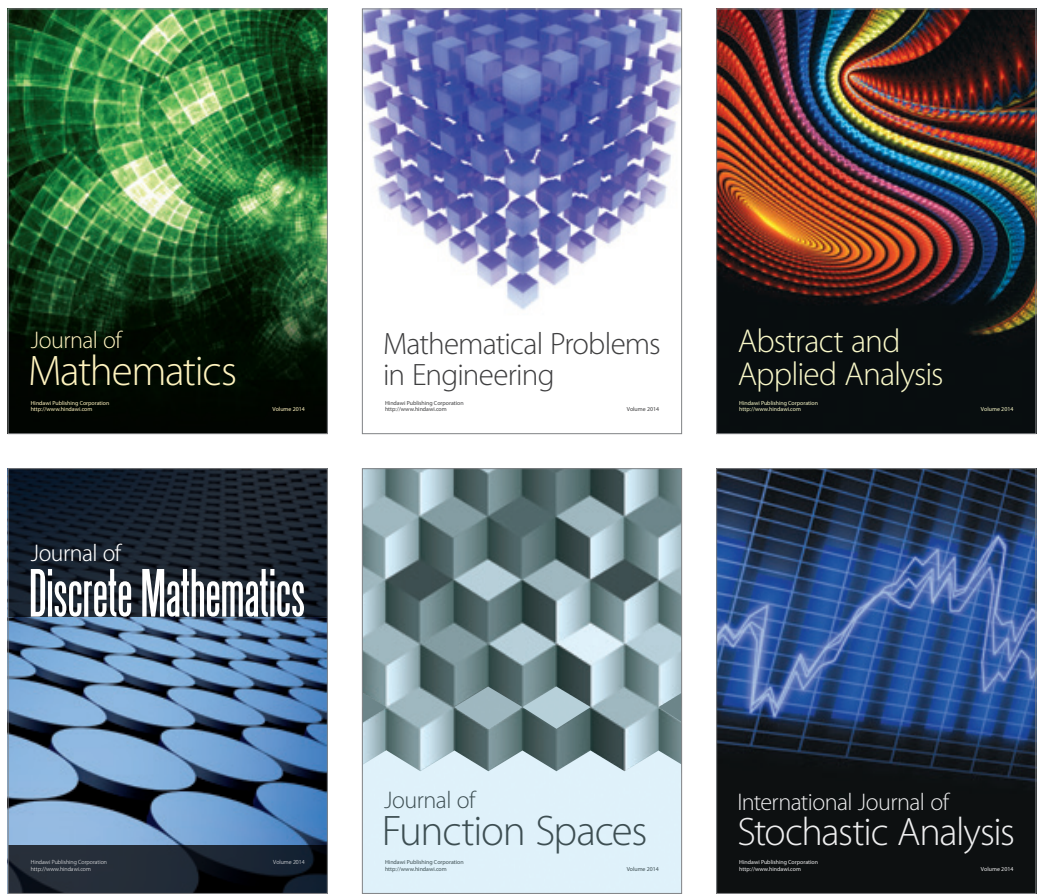

Journal of

Function Spaces

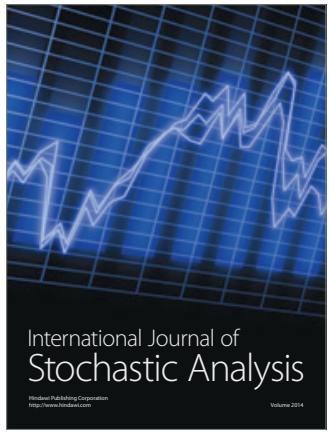

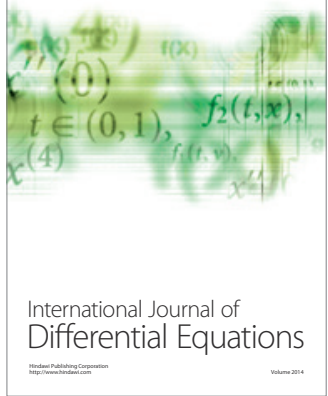
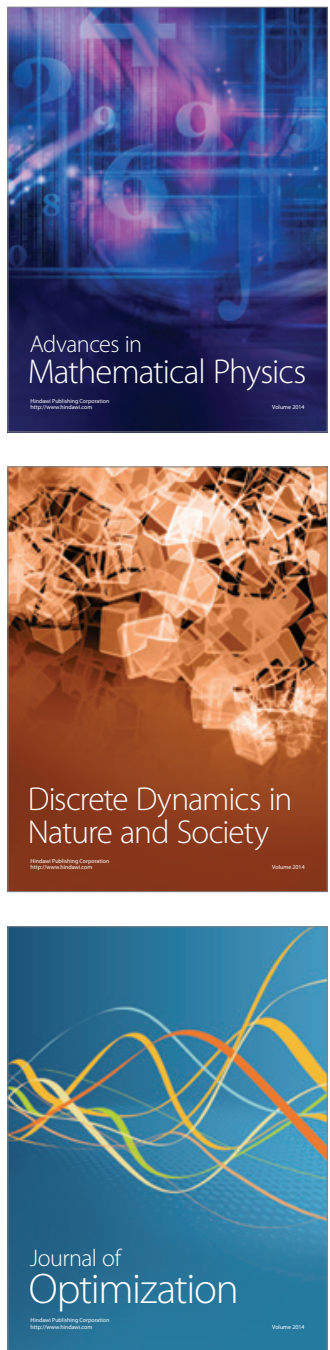